\title{
DETAILLED STUDY OF THE DYNAMICS \\ OF FRAGMENTS OF COMET C/1996 B2 HYAKUTAKE
}

\author{
E. DESVOIVRES AND J. KLINGER \\ Laboratoire de Glaciologie et de Géophysique de l'Environnement, \\ UJF-CNRS, 54, rue Molière, BP 96, Saint Martin d' Heres, France
}

and

\section{A.C. LEVASSEUR-REGOURD}

Université Paris 6, Service d'Aéronomie, CNRS, BP 3, 91371 Verrières, France

The fragmentation of cometary nuclei is a frequent phenomenon, but the dynamics of the fragments is not yet well understood. During the close approach of comet C/1996 B2 Hyakutake to the Earth (0.1 AU) on late March 1996, images were taken with the 1 meter telescope of Pic du Midi observatory. Bright condensations were observed near the nucleus on images taken between March, 22, 1996 and March, 31, 1996. It was suggested that these features were mini-coma surrounding fragments receding from the nucleus (Lecacheux et al., 1996). A model was developped for the motion of cometary fragments in the orbital plane of the comet, and the simulations were compared with the observations (Desvoivres et al, 1998).

In the model, we consider that the nucleus of the comet and a fragment are under the influence of the gravity of the Sun, of their mutual gravity, and of nongravitational forces (NGF) due the loss of mass induced by solar heating. From an estimation of those NGF, we compute numerically the trajectories of the fragment and of the nucleus with respect to their common center of mass (CoM). Then, the motion of the center of mass is studied in an heliocentric reference frame using the theory of perturbed keplerian motion.

The energy balance at the cometary surface is:

$$
S(1-a l)=\epsilon \sigma T^{4}+L_{I} \dot{Z}_{I}(T)
$$

where $S$ is the solar flux, al the albedo, $\epsilon$ the emissivity, $L_{I}$ the latent heat of sublimation of the water ice, and $\dot{Z}_{I}(T)$ the production rate of water vapour. The surface temperature is deduced from equation 1 by an iterative method. The value of the NGF is derived using the conservation of momentum.

The vectorial sum of the gravity and of the NGF is integrated numerically in order to obtain the momentum equation of both the nucleus and a fragment in the reference frame of the CoM. The modification of the motion of the CoM around the Sun is described using the Gauss equations (Danjon, 1959).

On several images, up to 4 fragments are visible at the same time. The evolution of the distance of the fragments to the nucleus as a function of time is known for each fragment. The values of the physical parameters are discussed in Desvoivres et al, 1998. It has been shown that the density and the size of the fragment are the only parameters which influence significantly the motion. Here, we have assumed 


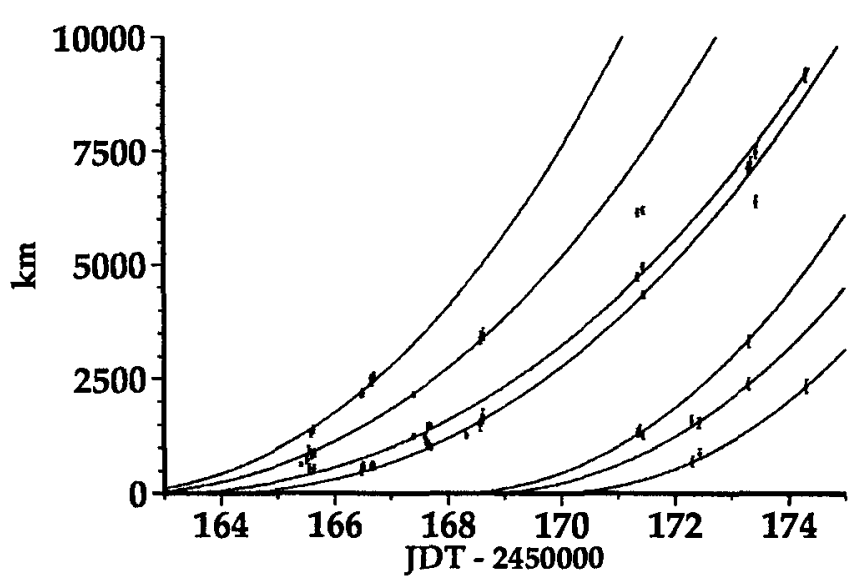

Fig. 1. Evolution of the distance to the nucleus of the simulated fragments (continuous lines), and comparison to the observations.

TABLE I

Characteristics of the different simulated fragments.

\begin{tabular}{|c|c|c|c|c|c|c|c|}
\hline Fragment & 1 & 2 & 3 & 4 & 5 & 6 & 7 \\
\hline Fragment size (meters) & 13 & 18 & 22 & 20 & 16 & 19 & 18 \\
\hline Emission date (March, 1996, TU) & 19.7 & 20.0 & 20.8 & 21.7 & 25.7 & 26.2 & 27.5 \\
\hline
\end{tabular}

the density to be constant and equal to $300 \mathrm{~kg} \cdot \mathrm{m}^{-3}$. Different numerical simulations were performed, and a $\chi^{2}$ method is used to select the best fit for each fragment. Seven different fragments are needed to reproduce the observations. The results are presented in figure 1 and the characteristics of the fragments are presented in table I. As can be noticed on figure 1, among 56 detected events, only 3 are not reproduced by the simulations. They might be due to fragmentation of fragments.

\section{Conclusion}

A model for the dynamics of cometary fragments was developped. It confirms the hypothesis that the bright condensations that were observed near comet C/1996 B2 Hyakutake are nucleus fragments. In agreement with the observations, the model shows that the fragments receed in the anti-solar direction.

\section{References}

Danjon A., 1959, Astronomie Générale, Albert Blanchart, Paris

E. Desvoivres, J. Klinger, A.C. Levasseur-Regourd, J. Lecacheux, L. Jorda, A. Enzian, F. Colas, E. Frappa, P. Laques, 1998, MN.R.A.S., accepted for publication.

Lecacheux J., Jorda L., Enzian A., Klinger J., Colas F., Frappa E., Laques P., 1996, IAU Circ. No 6354 